\title{
Concentration of clobetasone butyrate in aqueous humour
}

\author{
S. C. DEBNATH AND A. B. RICHARDS \\ From the Department of Ophthalmology, Royal Berkshire Hospital, Reading
}

\begin{abstract}
SUMMARY The concentrations of clobetasone butyrate and betamethasone were measured in aqueous humour of patients undergoing cataract extraction $12 \cdot 5$ to $18 \cdot 5$ hours after application into the lower conjunctival sac of an ointment containing $0 \cdot 1 \%$ of the steroid. Samples were assayed from 10 patients receiving clobetasone butyrate and 13 patients receiving betamethasone phosphate. There were measurable concentrations in only 2 samples in the former group, and both were $0 \cdot 1 \mathrm{ng} / \mathrm{ml}$. In the betamethasone group measurable concentrations were found in 11 samples, and the concentrations ranged from 0.5 to $20.3 \mathrm{ng} / \mathrm{ml}$, with the highest concentrations between 12.5 and 13.5 hours after application. The concentration of betamethasone in the aqueous humour decreased by about $90 \%$ in the 6 hours from 12.5 to 18.5 hours after application. It is speculative as to whether it is these differences in pharmacokinetic behaviour, or other differences in biological or physicochemical properties, which are responsible for the minimal effect on intraocular pressure induced by clobetasone butyrate compared with betamethasone.
\end{abstract}

Clobetasone butyrate $0 \cdot 1 \%$ (Eumovate) formulated as eye drops has been shown to be a safe, effective, topical steroid preparation in the treatment of steroid-responsive eye conditions. ${ }^{1-3}$ Its therapeutic effects have been shown to be equivalent to that of the well established betamethasone phosphate $0.1 \%$ (Betnesol). Several workers have shown that the propensity of clobetasone butyrate eye drops to raise intraocular pressure is less than is experienced with other topical corticosteroid eye preparations. ${ }^{2}$ The mechanism, by which intraocular pressure is raised by corticosteroids is not fully understood, so that the site at which the steroid is acting is not known. Whatever the mechanism it is possible that the concentration of the corticosteroid in the aqueous humour is an important determinant. We decided to investigate the concentration of clobetasone butyrate in the aqueous humour of patients undergoing cataract extraction and to compare these results with those obtained with betamethasone phosphate.

\section{Patients and methods}

Patients with a diagnosis of senile cataract who had been admitted to hospital just before surgery had the

Correspondence to Mr S. C. Debnath, FRCS. Alkharaj Military Hospital. PO Box 7897 Riyadh 11159, Saudi Arabia. nature of the study explained to them and their consent sought. They were then allocated, according to a randomisation code, to receive a single application of either clobetasone butyrate $0.1 \%$ or betamethasone phosphate $0 \cdot 1 \%$ eye ointment. A quarterinch $(5 \mathrm{~mm})$ extrusion of the ointment was placed under the lower fornix of the eye on the evening before surgery. The time was noted. The steroid ointments were prepared in the same bases and this method of administration had been shown previously to deliver a similar amount of the 2 steroids.

During operation the conjunctiva was cleaned and a sample of aqueous humour was collected by needle aspiration of the anterior chamber before incising the cornea. The specimen was placed in a plastic tube and stored at $-20^{\circ} \mathrm{C}$ until assay. The time of collection of the specimen was noted. The concentrations of the steroids in the samples of aqueous humour were measured by a radioimmunoassay technique. The antibodies used were specific for clobetasone butyrate and betamethasone respectively. The limits of assay of both steroids were $0 \cdot 1 \mathrm{ng} / \mathrm{ml}$. The method for betamethasone was unlikely to detect significant amounts of betamethasone phosphate as the degree of cross-reaction was $0 \cdot 1 \%$. There is evidence that the phosphate rapidly changes to the parent betamethasone. 
Table 1 Concentrations of clobetasone butyrate and betamethasone in aqueous humour at different times after application as ointments

\begin{tabular}{llc}
\hline $\begin{array}{l}\text { Treatment time } \\
\text { (hours before } \\
\text { surgery) }\end{array}$ & $\begin{array}{l}\text { Clobetasone butyrate } \\
\text { concentration } \\
(\mathrm{ng} / \mathrm{ml})\end{array}$ & $\begin{array}{l}\text { Betamethasone } \\
\text { concentration } \\
(\mathrm{ng} / \mathrm{ml})\end{array}$ \\
\hline $12 \cdot 5$ & $0 \cdot 1$ & $5 \cdot 3$ \\
$13 \cdot 0$ & $\mathrm{ND}$ & $2 \cdot 0$ \\
& & $1 \cdot 5$ \\
& & $6 \cdot 0$ \\
$13 \cdot 5$ & $\mathrm{ND}$ & $12 \cdot 5$ \\
$14 \cdot 0$ & $0 \cdot 1$ & $2 \cdot 5$ \\
$15 \cdot 0$ & $\mathrm{ND}$ & $20 \cdot 3$ \\
$16 \cdot 5$ & & \\
$17 \cdot 5$ & $\mathrm{ND}$ & $1 \cdot 0$ \\
& $\mathrm{ND}$ & $\mathrm{ND}$ \\
& $\mathrm{ND}$ & $\mathrm{ND}$ \\
18.0 & ND & \\
$18 \cdot 5$ & ND & $1 \cdot 5$ \\
\hline & & $0 \cdot 8$ \\
\hline
\end{tabular}

$\mathrm{ND}=$ none detected.

\section{Results}

Thirty patients were admitted to the study, 15 in each treatment group. In the clobetasone butyrate group there were 4 males and 11 females of mean age 80 years (range 56-91 years), and specimens of aqueous humour were collected between 12.5 and 18 hours after application of the ointment. In the betamethasone phosphate group there were 6 males and 9 females of mean age 75 years (range 51-86 years), and aqueous humour was collected between 12.5 and $18 \cdot 5$ hours after application of the ointment. Thus both groups were similar with respect to these factors.

Assay results were available from 10 patients in the clobetasone group and from 13 patients in the betamethasone group. Samples from the other 7 patients could not be assayed owing to an insufficient volume of aqueous humour. In the clobetasone butyrate group steroid was detected in only 2 samples of aqueous humour with a maximum concentration of $0 \cdot 1 \mathrm{ng} / \mathrm{ml}$. In the betamethasone phosphate group steroid was detected in 11 samples with the maximum concentration being $20 \cdot 3 \mathrm{ng} / \mathrm{ml}$ (Table 1 ). The mean concentration of betamethasone phosphate was $4 \cdot 1$ $\mathrm{ng} / \mathrm{ml}$. In the period between 12.5 and 13.5 hours after application 6 of the 7 samples containing betamethasone had higher concentrations than any of the 6 samples collected between $16 \cdot 5$ and 18.5 hours after application. The lowest concentration in the earlier time period $(1.5 \mathrm{ng} / \mathrm{ml})$ was exactly the same as the highest concentration in the later time period. Although it is difficult to calculate pharmacokinetic indices when single samples have been collected from a number of patients, it would appear that in the 6 hours between 12.5 and 18.5 hours after application the concentration of betamethasone in the aqueous humour decreased by about $90 \%$. It is not possible to do similar calculations with clobetasone butyrate, as there were only 2 samples in which the drug was detected, and the concentrations were the same.

\section{Discussion}

Clobetasone butyrate and betamethasone phosphate are both glucocorticosteroids, but their biological properties and physicochemical characteristics differ. In the vasoconstrictor test, which is a method of ranking topical activity, clobetasone butyrate is much more potent than betamethasone ( 250 compared with $1 \cdot 0)$. Thus, if topical activity is important in the treatment of external and internal inflammatory conditions of the eye, a therapeutic effect will be achieved with a lower concentration of clobetasone butyrate. It has been shown that clobetasone butyrate is therapeutically effective in both superficial conditions and also internal conditions such as uveitis. Despite being clinically effective, clobetasone butyrate seems to have.little, if any, effect on intraocular pressure compared with other corticosteroids. This must mean that the site at which corticosteroids increase intraocular pressure is inaccessible to clobetasone butyrate or the receptor is poorly responsive or nonresponsive. It would be interesting to compare these results with the concentrations of other corticosteroids known to raise intraocular pressure.

The very low concentrations of clobetasone butyrate found in this study were obtained after application of the steroid in an ointment base. With this vehicle an increased time of contact of the steroid with the eye is achieved compared with application as eye drops. It has been thought that higher concentrations of drugs are obtained in the various compartments of the eye with ointments than with drops.

The solubilities of these 2 steroids are quite different. Clobetasone butyrate is almost insoluble in water $(0.5$ parts per million) whereas betamethasone phosphate is highly soluble $(20 \%)$. In contrast clobetasone butyrate is much more lipid soluble. The partition ratio between water and cyclohexane is an estimate of lipid solubility and is $1: 3 \cdot 0$ for clobetasone butyrate and 1:0.02 for betamethasone phosphate. The parent betamethasone, which was measured in the aqueous humour, has a partition ratio similar to that for the phosphate derivative, namely, 1:0.03. From these data it seems that clobetasone butyrate is much more likely to be absorbed into the cells of the cornea and the conjunctiva than is betamethasone phosphate. Moreover, its concentration in the cells 
will increase, as relatively little will diffuse into the aqueous humour. The partition coefficient of betamethasone on the other hand indicates that this steroid is likely to diffuse into the aqueous humour more readily.

The collection of samples in this study was made between 12.5 and 18.5 hours after application. It is of course possible that in the period up to 12.5 hours the concentration of clobetasone butyrate had already reached a peak and declined rapidly. In a similar way we do not know whether betamethasone reached its peak concentration in the period under study or earlier. Further experiments are required to answer these questions.

In conclusion, this study has shown that the pharmacokinetics of clobetasone butyrate and betamethasone after application to the human eye in an ointment formulations are different. It has previously been shown that both steroids, at least in an eye drop formulation, have similar clinical efficacy in the treatment of external and internal inflammatory conditions of the eye. However, they do differ in their propensity to increase intraocular pressure, in their physicochemical properties, and in their topical activity. Which, if any, of these differences is the most important for the lack of effect of clobetasone butyrate upon intraocular pressure is not known, but the difference in pharmacokinetics could either be the final common pathway or a crucial link in the chain.

We are grateful to the Drug Metabolism Department of Glaxo Group Research Ltd, Greenford, for assaying the concentrations of clobetasone butyrate and betamethasone. We are also grateful to all the consultants at the Royal Berkshire Hospital for permitting us to do this research work on their patients.

\section{References}

1 Dunne JA, Travers JP. Double-blind clinical trial of topical steroids in anterior uveitis. Br J Ophthalmol 1979; 63: 762-7.

2 Eilon LA, Walker SR. The clinical evaluation of clobetasone butyrate in the treatment of anterior uveitis. $\mathrm{Br} \mathrm{J} \mathrm{Ophthalmol}$ 1981; 65: 644-7.

3 Ramsell TG, Bartholomew RS, Walker SR. Clinical evaluation of clobetasone butyrate: a comparative study of its effect in postoperative inflammation and on intraocular pressure. $\mathrm{Br} J$ Ophthalmol 1980; 64: 43-5. 